\title{
Immunotherapy of Cancer: Reprogramming Tumor-Immune Crosstalk
}

\author{
Kyle K. Payne, ${ }^{1}$ Amir A. Toor, ${ }^{2}$ Xiang-Yang Wang, ${ }^{3}$ and Masoud H. Manjilii ${ }^{1}$ \\ ${ }^{1}$ Department of Microbiology \& Immunology, Massey Cancer Center, Virginia Commonwealth University, Richmond, \\ VA 23298, USA \\ ${ }^{2}$ Department of Internal Medicine, Massey Cancer Center, Virginia Commonwealth University, Richmond, VA 23298, USA \\ ${ }^{3}$ Department of Human and Molecular Genetics, Massey Cancer Center, Virginia Commonwealth University, Richmond, \\ VA 23298, USA
}

Correspondence should be addressed to Masoud H. Manjili, mmanjili@vcu.edu

Received 2 August 2012; Accepted 25 September 2012

Academic Editor: Guido Kroemer

Copyright (๑) 2012 Kyle K. Payne et al. This is an open access article distributed under the Creative Commons Attribution License, which permits unrestricted use, distribution, and reproduction in any medium, provided the original work is properly cited.

\begin{abstract}
The advancement of cancer immunotherapy faces barriers which limit its efficacy. These include weak immunogenicity of the tumor, as well as immunosuppressive mechanisms which prevent effective antitumor immune responses. Recent studies suggest that aberrant expression of cancer testis antigens (CTAs) can generate robust antitumor immune responses, which implicates CTAs as potential targets for immunotherapy. However, the heterogeneity of tumor cells in the presence and quantity of CTA expression results in tumor escape from CTA-specific immune responses. Thus, the ability to modulate the tumor cell epigenome to homogenously induce expression of such antigens will likely render the tumor more immunogenic. Additionally, emerging studies suggest that suppression of antitumor immune responses may be overcome by reprogramming innate and adaptive immune cells. Therefore, this paper discusses recent studies which address barriers to successful cancer immunotherapy and proposes a strategy of modulation of tumor-immune cell crosstalk to improve responses in carcinoma patients.
\end{abstract}

\section{Introduction}

Conventional approaches in the therapy of cancer, such as chemotherapy, have shown only modest success in the treatment of advanced carcinoma [1]. Historical comparisons since the late 1970s have shown that the introduction of combination cytotoxic chemotherapy has produced a modest 9-12 month gain in survival compared with untreated breast cancer patients [2]. Despite advances in conventional cytotoxic therapies of early-stage breast cancer $[3,4]$ there remains no therapeutic strategy that can ensure relapsefree survival. Furthermore, studies have shown that 20\% of clinically disease-free early-stage breast cancer patients relapse within 10 years after conventional therapies [5]; indeed, most cancer-related deaths within the United States are attributed to relapse [6]. Thus, there is an urgent need to develop more effective therapies to overcome breast cancer relapse and to treat advanced cancer. To this end, immunotherapy emerges as promising strategy for the prevention of tumor relapse, when combined with conventional therapies.

Thus far advances in the immunotherapy of cancer have also been met with a number of setbacks. Several vaccination strategies used against breast cancer have been successfully employed to induce tumor-specific $\mathrm{CD}^{+}$and $\mathrm{CD}^{+} \mathrm{T}$ cell responses; however, such immunological responses have rarely been potent enough to achieve objective results [7-9]. Additionally, it has been demonstrated by several groups that adoptive cellular therapy (ACT) directed against highly immunogenic melanoma-associated antigens results in objective responses in animal models as well as in some melanoma patients $[10,11]$. ACT has also been tested against breast cancer both in preclinical and clinical studies [12, 13]; however, unlike melanoma, ACT has not produced promising results in breast cancer patients and has only displayed effectiveness in animal models in prophylactic settings $[14,15]$, rather than against well-established, vascularized tumors. Such failure has been attributed, in part, to (i) the 
lack of a robust antitumor immune response as a result of the expression of weakly immunogenic tumor antigens coupled with the presence of low frequency and low affinity $\mathrm{T}$ cells and (ii) the suppression of antitumor immune responses though the activity of immunosuppressive mechanisms. Indeed, distant recurrence of breast cancer may occur even in the presence of tumor-specific immune responses. The ability to overcome these barriers will likely improve the efficacy of immunotherapy directed against cancer. To address these issues, the crosstalk between tumor cells and cells of the immune system should be altered in order for reprogrammed tumor cells and immune cells to prevent tumor relapse as well as induce regression of advanced cancer.

\section{Immune Suppression}

It is now well established that the mammalian immune response can be suppressed through various mechanisms. The expression of immunoregulatory molecules, such as CTLA-4 and PD-1 as well as the ectoenzyme, CD73, inhibits the proliferation and function of conventional $\mathrm{T}$ cells $[16,17]$. Furthermore, immunosuppressive cells such as alternatively activated M2 macrophages, type II NK cells, and regulatory $\mathrm{T}$ cells have been demonstrated to antagonize tumor immunosurveillance [18-22].

Results from clinical studies involving breast cancer patients indicate that another critical regulator of tumor immunosurveillance, the myeloid-derived suppressor cell (MDSC), was found to be the most abundant type of suppressor cell $[23,24]$ and thus represent a major hurdle in overcoming antitumor immune suppression. MDSCs represent a phenotypically heterogeneous population of myeloid cells at different stages of maturation. These cells have been found in tumor-bearing mice as well as cancer patients and have been shown to possess multiple mechanisms to suppress the antitumor immune response $[25,26]$. Such responses include disrupting TCR antigen recognition and T-cell mediated IFN- $\gamma$ production $[27,28]$, depletion of essential amino acids within the tumor microenvironment [29], and overproduction of reactive oxygen species (ROS) [30]. Murine MDSCs are defined as coexpressing Gr- 1 and CD11b, with two subsets commonly being described: granulocytic $\left(\mathrm{CD} 11 \mathrm{~b}^{+} \mathrm{Ly}-6 \mathrm{G}^{+} \mathrm{Ly}-6 \mathrm{C}^{\text {low }}\right)$ and monocytic $\left(\mathrm{CD} 11 \mathrm{~b}^{+}\right.$Ly$6 \mathrm{G}^{-}$Ly-6C $\left.\mathrm{C}^{\text {high }}\right)$ [31]. Human MDSCs, on the other hand, have been difficult to be identified as initial studies revealed that these cells express varied phenotypes and suppressive patterns [25]. It is now regarded, however, that human MDSCs fall into two main subsets: a monocytic population characterized by expression of CD14 and a granulocytic population characterized by CD15 expression; both subtypes have been reported to express the common myeloid markers $\mathrm{CD} 11 \mathrm{~b}$ and CD33, with minimal expression of myeloid maturation markers such as HLA-DR [32]. The accumulation of these cells in association with cancer development is corroborated by experimental mouse models, indicating that MDSCs develop as a function of tumor progression [33]. For instance, our group has previously reported that FVBN202 mice, which overexpress the rat neu oncogene in their mammary glands, develop atypical ductal hyperplasia $(\mathrm{ADH})$ and ductal carcinoma in situ (DCIS) in mammary epithelial cells prior to the formation of spontaneous mammary tumors [34]. DCIS of the breast is conventionally regarded as a precursor of invasive breast cancer, and $\mathrm{ADH}$ is a risk factor for the development of the disease [35, 36]. Compromised antineu immune responses occur as a result of the emergence of premalignant events, such as ADH and DCIS, which are characterized by an accumulation of MDSCs in the blood, bone marrow, secondary lymphoid tissues, and within tumor lesions due to an increased production of tumor-derived soluble factors [34, 37-41]. Such findings provide evidence that MDSCs function as potent inhibitors of antitumor immunity in breast cancer models. Likewise, human MDSCs have been observed to negatively regulate both adaptive and innate immunity during cancer development and progression, with accumulation having been observed in peripheral blood and lymphoid tissues as well as draining tumor sites of cancer-bearing patients [31]. In addition to breast cancer, the accumulation of MDSCs has been observed in other neoplastic diseases, such as hepatocellular, pancreatic, esophageal, and colorectal cancers [26], and is generally correlated with advanced clinical cancer stage and metastatic tumor burden with a demonstrated suppression of antitumor immune responses correlating with poor responses following conventional therapies [14, 23, 42, 43]. Thus, MDSC accumulation is paramount in the ability of cancer to evade effective immune responses. Therefore, suppression of immune responses mediated by MDSCs must be overcome to rescue and facilitate effective tumor-specific immunity. Accordingly, it was reported that activated NKT cells can overcome MDSCs, thereby supporting an effective adaptive immune response against cancer $[15,44,45]$. Our group has recently developed a novel strategy of reprogramming immune cells ex vivo to overcome MDSC-mediated antitumor immune suppression in a prophylactic model of murine breast carcinoma upon adoptive transfer, which resulted in a demonstrated ability to enhance immune mediated rejection of tumors [15]. However, this approach failed to protect mice in a therapeutic model against established tumors. Thus, in addition to overcoming MDSC-mediated suppression, improvements in the efficacy of immunotherapy likely will require further addressing the crosstalk between immune and tumor cells; one such strategy is enhancing tumor cell immunogenicity.

\section{In Situ Vaccination: Modulating the Tumor Cell Epigenome}

A barrier for successful immunotherapy of breast cancer is the low immunogenicity of tumor cells, for example, expression of tumor associated antigens which are recognized as "self" by the immune system. Therefore, improving the immunogenicity of tumor is essential to improving tumor immunotherapy. To this end, in situ induction of foreign-like antigens, such as cancer testis antigens (CTA), to which Tcell tolerance does not exist, is a promising option. CTAs are 
highly immunogenic with no natural self-tolerance due to the observation that they are normally only expressed during embryonic development; after birth, expression is generally limited to immunologically privileged germ cells and the placenta [46]. Aberrant CTA expression was first described in melanoma; as such, this expression was found to generate CTA-specific cytotoxic T-cell responses [47]. Recently, it was reported that treatment of metastatic melanoma with autologous $\mathrm{CD}^{+}{ }^{+} \mathrm{T}$ cells specific for the CTA, NY-ESO1, elicited long-term complete remission [48]. In addition to melanoma, CTA expression has also been observed in hematological malignancies [49] as well as solid tumors, including breast cancer $[50,51]$. Further, CTA expression in breast cancer has been shown to elicit a broad range of cellular and humoral immune responses [50, 52, 53]; both $\mathrm{CD}^{+}{ }^{+} \mathrm{T}$ cell and $\mathrm{CD} 79^{+} \mathrm{B}$ cell infiltration has been observed in primary and metastatic NY-ESO-1 expressing breast cancer [54]. Of note, a significantly elevated expression of NY-ESO-1 and MAGE-A, another highly immunogenic CTA, was detected in triple negative breast cancers compared to other types of breast cancer [55], which therefore represent antigenic targets in an otherwise immunologically refractory breast cancer subtype.

Importantly, CTA expression is normally silenced by methylation within the promoter region of these genes. Methylation at the C-5 position of cytosine bases within DNA is a covalent chemical modification which characterizes a key, biologically functional, epigenetic modification of the animal genome [56]. This action primarily occurs at $\mathrm{CpG}$ dinucleotides in mammals, where DNA-methyltransferases (DNMTs) mediate the transfer of methyl groups to cytosine, thereby generating 5-methylcytosine $(5 \mathrm{mC})$ that has been shown to play a critical role in the cellular protein expression by transcriptional silencing of genes [57]. Aberrant CTA expression likely occurs due to epigenetic molecular alterations which arise during tumor progression; cancer cells display drastic changes in DNA methylation status, typically exhibiting global DNA hypomethylation as well as region-specific hypermethylation [58], resulting in irregular expression of CTAs. Our group has observed that a lack of such aberrant CTA expression within breast tumor lesions at the time of diagnosis correlated with eventual relapse after conventional therapies (unpublished data) along with the lack of expression of an immune function gene signature [59]. Conversely, the tumors in patients who remained free of relapse expressed both CTAs and the immune function gene signature. These data suggest that CTA expression in breast cancer patients activates effective immune responses which results in improved prognosis after conventional treatments.

In order to induce and/or increase expression of CTAs to function as target antigens and improve the prognosis in patients with breast cancer, it is possible to modulate the tumor epigenome to initiate the cellular CTA transcriptional program; such an approach will serve to impart a more immunogenic tumor cell phenotype. Azacitidine (Aza) and Decitabine (Dec) are both hypomethylating agents employed in epigenetic therapy to modify cellular methylation patterns; both of these agents have been approved for clinical use in the treatment of myelodysplastic syndrome. Aza and
Dec function as cytosine analogs, which lead to their incorporation into newly synthesized DNA strands during $S$ phase of the cell cycle; these agents have been shown to induce and/or increase the expression of various CTAs in a variety of in vitro and in vivo tumor models [49, 52-54]. Both Aza and Dec have demonstrated the ability to induce the expression of CTAs, as well as the tumor suppressor gene p53 [60] and the death receptor Fas [61] on tumor cells. These are attributed to their capacity to function as potent DNMT inhibitors through the formation of a covalent complex with a serine residue at the active site of DNMT1, which therefore results in CpG island demethylation during cellular proliferation. This, in turn, results in hypomethylation within the promoter of tumor suppressor genes as well as a highly immunogenic CTAs [56, 62-64], thereby rendering tumor cells susceptible to CTAreactive immune responses and suppression of proliferation via expression of $\mathrm{p} 53$, as well as rendering these tumor cells more susceptible to Fas L-induced apoptosis by CTA-reactive $\mathrm{T}$ cells. Such modulation of CTA expression using Aza has been shown to generate CTA-specific T-cell responses in patients with acute myeloid leukemia, as demonstrated by our group [65]. Others have demonstrated the feasibility to induce CTA expression in vivo using Dec in the 4T1 model of murine breast carcinoma, resulting in greater tumor cell cytotoxicity upon treatment with CTA-specific T cells [56]. Further, an ongoing clinical trial in breast cancer patients is testing the efficacy of Dec for the induction of the expression of ER/PR in patients with hormone receptor negative tumors in order to render them susceptible to hormonal therapy [66].

Decitabine is a particularly attractive option to induce CTA expression as it functions as a prodrug which requires activation by deoxycytidine kinase (DCK), an enzyme preferentially expressed in tumor cells and myeloid cells. Thus, the effects of Dec are likely tissue specific, as DCK is selectively expressed in tumor cells and myeloid cells, thus protecting $\mathrm{T}$ and $\mathrm{B}$ cells from the potentially deleterious demethylating effects of this agent. In addition, DCK has been found to be overexpressed in poor outcome breast cancer [67], suggesting that epigenetic therapy to induce CTA expression may prove to be an efficacious approach in breast cancer patients with poor prognosis.

Our group has recently demonstrated that epigenetic modulation using sequential Aza and the immunomodulatory agent lenalidomide for the induction of CTA expression in the tumor and CTA-specific antitumor immune responses in patients with multiple myeloma [65]. Upon determination of CTA expression in bone marrow of multiple myeloma patients following treatment with Aza, we found that CTA expression is induced exclusively in $\mathrm{CD} 138^{+}$malignant plasma cells in vivo, which suggests a preferential induction of hypomethylation in CTA promoters within tumor cells. As a result of such a strategy, which we term in situ vaccination or epigenetic induction of an adaptive immune response, we have determined that the observed induction of CTA expression resulted in the generation of robust CTAspecific adaptive immune responses [65]. We believe that this strategy will maintain long-term surveillance against 
malignant plasma cells in patients with $\mathrm{MM}$ and translate into prolonged freedom from progression in this otherwise incurable disease. Furthermore, these data suggest that epigenetic therapeutic agents, such as Dec, when used in a neoadjuvant setting, may induce CTA expression in tumor-bearing patients and may therefore activate early CTA-specific immune responses to prevent recurrence after conventional therapies.

\section{Reprogramming of Tumor-Sensitized Immune Cells}

The rationale for ex vivo reprogramming of tumor-sensitized immune cells is based on overcoming the low frequency of endogenous tumor-reactive $\mathrm{T}$ cells by driving their expansion and activation toward the most effective antitumor phenotype(s). We have previously shown the ability of ex vivo reprogrammed Her $2 /$ neu sensitized immune cells to protect mice in a prophylactic setting when used in an adoptive cellular therapy (ACT) setting [15]. Cellular reprogramming through the combined use of bryostatin 1, a potent activator of classical and novel protein kinase $\mathrm{C}$ (PKC) $[68,69]$, and ionomycin (B/I), a calcium ionophore $[70,71]$, followed by differentiation using gamma-chain $(\gamma$ c) cytokines (IL-2, IL-7, and IL-15) results in the ability to selectively activate tumor-primed T cells, NK cells, and NKT cells, as described by our group $[72,73]$. In particular, the generation of both $\mathrm{CD}^{+}$and $\mathrm{CD}^{+}$central memory $\left(\mathrm{CD} 44^{+} \mathrm{CD}^{2} \mathrm{~L}^{\text {high }}\right)$ lymphocytes, which are necessary to mediate protection in ACT recipients upon challenge with antigen expressing tumor cells, is observed. Furthermore, we observed that reprogrammed NK/NKT cells surprisingly functioned to render $\mathrm{T}$ cells resistant to MDSC suppression and induced tumor rejection even in the presence of MDSC in FVBN202 mice [15].

Therefore, it may prove beneficial to harvest autologous peripheral blood mononuclear cells (PBMC) from breast cancer patients having received neoadjuvant Dec treatment in order to reprogram CTA-sensitized immune cells using $\mathrm{B} / \mathrm{I}$ and $\gamma$-c cytokines; following conventional therapies, such reprogrammed lymphocytes can then be reinfused back into the host, whereupon they may exert long-lived protection against relapse, even in the presence of classical immunosuppressive cells such as MDSCs.

\section{Rescue of Late Antitumor Immune Responses}

We have previously demonstrated that MDSC accumulation results as a function of tumor-derived soluble factors, such as GM-CSF, in the FVBN202 model of breast carcinoma [74], while others have identified additional tumor-derived soluble factors and inflammatory cytokines which are responsible for the accumulation of MDSCs [38-41]. We have also verified the ability of radiation therapy (RT) to reject primary tumors, thereby resulting in the reduction of MDSCs within the tumor bearing host [15]. Ionizing irradiation is known to cause cellular stress and enhance the synthesis of a variety of immune-stimulatory and modulating molecules such as heat shock proteins (HSP) $[75,76]$, high mobility group box 1 (HMGB1) [76], and NKG2D ligands [77]. Such danger signals are then sensed by cells of the immune system. For instance, toll-like receptor (TLR)-4 on DCs interacts with its ligands including HMGB1 [78] and HSPs [79] and enhances maturation and antigen presentation capacity of DCs. Detection of danger signals in tissues by leukocytes activates an immune response involving cells of the innate (myeloid and NK cells) and adaptive ( $\mathrm{T}$ and $\mathrm{B}$ cell) lineages. RT-induced NKG2D ligand, an activating receptor for NK cells, and HSP70 render tumor cells more susceptible to NK-cell-mediated cytolysis [80]. Thus, combining RT with an enhanced immunotherapeutic strategy, such as neoadjuvant administration of Dec, is likely to enhance antitumor immune responses and produce objective responses against advanced breast cancer and result in a decreased risk of disease relapse. The removal of MDSCmediated suppression via RT may, therefore, facilitate the rescue of CTA-specific antitumor immune responses against residual tumor cells and result in the prevention of future disease recurrence. Accordingly, we propose that CTA-reactive $\mathrm{T}$ cells became antigen experienced during tumorigenesis due to aberrant CTA expression; however, it is likely that such CTA expression occurs late in the progression of the tumor, thus rendering CTA-reactive T cells ineffective due to MDSC accumulation via tumor-derived soluble factors. It is expected, nevertheless, that patients who receive neoadjuvant Dec followed by radiation therapy or surgery to remove the primary tumor will experience a reduction in MDSC accumulation; we propose that such activity will result in the rescue of CTA-reactive $\mathrm{T}$ cells from suppression to eliminate residual tumor cells in order to decrease the likelihood of future disease recurrence.

\section{Limitations and Future Considerations}

The majority of solid tumors and hematological malignancies undergo a period of dormancy that is characterized by years to decades of minimal residual disease (MRD) in which cancer progression has paused [81, 82]. Indeed, diseasefree periods in breast cancer patients can last as long as 25 years and are clearly associated with the presence of MRD; subsequent relapse represents the escape of the tumor from dormancy, which can include locoregional recurrence as well as distant metastatic disease [81, 83, 84]. Tumor dormancy may be the result of hypoxic stress, as well as other as yet unknown cues from the microenvironment of the host [85]. The mechanism of tumor cell dormancy may best be explained by cellular quiescence. Quiescence is defined as growth/proliferation arrest and is thought to be due to G0G1 cell cycle arrest, during which cells pause cellular activities which can render them refractory to differentiation and proliferation $[86,87]$. Thus, given that DNMT inhibitors Dec and Aza are incorporated into cellular DNA during $S$ phase, the induction of CTA expression requires tumor cells to be actively proliferating. As such, the in situ vaccination strategy outlined above will likely be less effective against any residual 
tumor cells that have entered G0-G1 arrest. Therefore, further understanding the process by which residual tumor cells naturally exit dormancy may provide novel approaches to coax such cells to exit cell-cycle arrest. Future studies investigating the ability of Aza or Dec combined with histone deacetylase inhibitors (HDI) to reinitiate the cell cycle would be beneficial in addressing this problem. Such efforts may result in an enhanced ability of in situ vaccination strategy to target and eliminate MRD, which may therefore lower the incidence of tumor recurrence presently observed in breast cancer patients.

\section{Acknowledgments}

The authors gratefully acknowledge the support of VCU Massey Cancer Center and the Commonwealth Foundation for Cancer Research.

\section{References}

[1] M. A. Cobleigh, "Other options in the treatment of advanced breast cancer," Seminars in Oncology, vol. 38, supplement 2, pp. S11-S16, 2011.

[2] S. R. Johnston, "The role of chemotherapy and targeted agents in patients with metastatic breast cancer," European Journal of Cancer, vol. 47, supplement 3, pp. S38-S47, 2011.

[3] J. Berry, "Are all aromatase inhibitors the same? A review of controlled clinical trials in breast cancer," Clinical Therapeutics, vol. 27, no. 11, pp. 1671-1684, 2005.

[4] C. J. Markopoulos, "Minimizing early relapse and maximizing treatment outcomes in hormone-sensitive postmenopausal breast cancer: efficacy review of AI trials," Cancer and Metastasis Reviews, vol. 29, no. 4, pp. 581-594, 2010.

[5] A. M. Brewster, G. N. Hortobagyi, K. R. Broglio et al., "Residual risk of breast cancer recurrence 5 years after adjuvant therapy," Journal of the National Cancer Institute, vol. 100, no. 16, pp. 1179-1183, 2008.

[6] J. R. Benson, I. Jatoi, M. Keisch, F. J. Esteva, A. Makris, and V. C. Jordan, "Early breast cancer," The Lancet, vol. 373, no. 9673, pp. 1463-1479, 2009.

[7] G. Curigliano, G. Spitaleri, E. Petri et al., "Breast cancer vaccines: a clinical reality or fairy tale?" Annals of Oncology, vol. 17, no. 5, pp. 750-762, 2006.

[8] J. Zhou and Y. Zhong, "Breast cancer immunotherapy," Cellular \& Molecular Immunology, vol. 1, no. 4, pp. 247-255, 2004.

[9] M. Z. Ladjemi, W. Jacot, T. Chardès, A. Pèlegrin, and I. Navarro-Teulon, "Anti-HER2 vaccines: new prospects for breast cancer therapy," Cancer Immunology, Immunotherapy, vol. 59, no. 9, pp. 1295-1312, 2010.

[10] A. G. Chapuis, J. A. Thompson, K. A. Margolin et al., "Transferred melanoma-specific $\mathrm{CD}^{+} \mathrm{T}$ cells persist, mediate tumor regression, and acquire central memory phenotype," Proceedings of the National Academy of Sciences of the United States of America, vol. 109, no. 12, pp. 4592-4597, 2012.

[11] H. K. Le, L. Graham, C. H. Miller, M. Kmieciak, M. H. Manjili, and H. D. Bear, "Incubation of antigen-sensitized T lymphocytes activated with bryostatin $1+$ ionomycin in IL-7 + IL-15 increases yield of cells capable of inducing regression of melanoma metastases compared to culture in IL-2," Cancer Immunology, Immunotherapy, vol. 58, no. 10, pp. 1565-1576, 2009.

[12] S. Tuve, B. M. Chen, Y. Liu et al., "Combination of tumor sitelocated CTL-associated antigen-4 blockade and systemic regulatory T-cell depletion induces tumor-destructive immune responses," Cancer Research, vol. 67, no. 12, pp. 5929-5939, 2007.

[13] H. Bernhard, J. Neudorfer, K. Gebhard et al., "Adoptive transfer of autologous, HER2-specific, cytotoxic T lymphocytes for the treatment of HER2-overexpressing breast cancer," Cancer Immunology, Immunotherapy, vol. 57, no. 2, pp. 271-280, 2008.

[14] J. K. Morales, M. Kmieciak, L. Graham, M. Feldmesser, H. D. Bear, and M. H. Manjili, "Adoptive transfer of HER2/neuspecific $\mathrm{T}$ cells expanded with alternating gamma chain cytokines mediate tumor regression when combined with the depletion of myeloid-derived suppressor cells," Cancer Immunology, Immunotherapy, vol. 58, no. 6, pp. 941-953, 2009.

[15] M. Kmieciak, D. Basu, K. K. Payne et al., "Activated NKT cells and NK cells render T cells resistant to myeloid-derived suppressor cells and result in an effective adoptive cellular therapy against breast cancer in the FVBN202 transgenic mouse," Journal of Immunology, vol. 187, no. 2, pp. 708-717, 2011.

[16] E. Y. Woo, C. S. Chu, T. J. Goletz et al., "Regulatory $\mathrm{CD} 4{ }^{+} \mathrm{CD} 25^{+} \mathrm{T}$ cells in tumors from patients with early-stage non-small cell lung cancer and late-stage ovarian cancer," Cancer Research, vol. 61, no. 12, pp. 4766-4772, 2001.

[17] J. Stagg, U. Divisekera, N. McLaughlin et al., "Anti-CD73 antibody therapy inhibits breast tumor growth and metastasis," Proceedings of the National Academy of Sciences of the United States of America, vol. 107, no. 4, pp. 1547-1552, 2010.

[18] C. Bergmann, L. Strauss, Y. Wang et al., "T regulatory type 1 cells in squamous cell carcinoma of the head and neck: mechanisms of suppression and expansion in advanced disease," Clinical Cancer Research, vol. 14, no. 12, pp. 37063715, 2008.

[19] A. M. Thornton and E. M. Shevach, "CD4 ${ }^{+} \mathrm{CD} 25^{+}$immunoregulatory $\mathrm{T}$ cells suppress polyclonal $\mathrm{T}$ cell activation in vitro by inhibiting interleukin 2 production," Journal of Experimental Medicine, vol. 188, no. 2, pp. 287-296, 1998.

[20] J. Shimizu, S. Yamazaki, and S. Sakaguchi, "Induction of tumor immunity by removing $\mathrm{CD} 25^{+} \mathrm{CD} 4^{+} \mathrm{T}$ cells: a common basis between tumor immunity and autoimmunity," Journal of Immunology, vol. 163, no. 10, pp. 5211-5218, 1999.

[21] M. R. Young, M. A. Wright, Y. Lozano et al., "Increased recurrence and metastasis in patients whose primary head and neck squamous cell carcinomas secreted granulocytemacrophage colony-stimulating factor and contained CD $34^{+}$ natural suppressor cells," International Journal of Cancer, vol. 74, pp. 69-74, 1997.

[22] L. Bingle, N. J. Brown, and C. E. Lewis, "The role of tumourassociated macrophages in tumour progression: implications for new anticancer therapies," Journal of Pathology, vol. 196, no. 3, pp. 254-265, 2002.

[23] C. M. Diaz-Montero, M. L. Salem, M. I. Nishimura, E. Garrett-Mayer, D. J. Cole, and A. J. Montero, "Increased circulating myeloid-derived suppressor cells correlate with clinical cancer stage, metastatic tumor burden, and doxorubicin-cyclophosphamide chemotherapy," Cancer Immunology, Immunotherapy, vol. 58, no. 1, pp. 49-59, 2009. 
[24] B. L. Mundy-Bosse, L. M. Thornton, H. C. Yang, B. L. Andersen, and W. E. Carson, "Psychological stress is associated with altered levels of myeloid-derived suppressor cells in breast cancer patients," Cellular Immunology, vol. 270, no. 1, pp. 8087, 2011.

[25] P. Filipazzi, V. Huber, and L. Rivoltini, "Phenotype, function and clinical implication of myeloid-derived suppressor cell in cancer patients," Cancer Immunology, Immunotherapy, vol. 61, pp. 255-263, 2012.

[26] S. Ohki, M. Shibata, K. Gonda et al., "Circulating myeloidderived suppressor cells are increased and correlate to immune suppression, inflammation and hypoproteinemia in patients with cancer," Oncology Reports, vol. 28, no. 2, pp. 453-458, 2012.

[27] M. Terabe, S. Matsui, J. M. Park et al., "Transforming growth factor- $\beta$ production and myeloid cells are an effector mechanism through Which CD1d-restricted T cells block cytotoxic $\mathrm{T}$ lymphocyte-mediated tumor immunosurveillance: abrogation prevents tumor recurrence," Journal of Experimental Medicine, vol. 198, no. 11, pp. 1741-1752, 2003.

[28] S. Kusmartsev, Y. Nefedova, D. Yoder, and D. I. Gabrilovich, "Antigen-specific inhibition of $\mathrm{CD}^{+} \mathrm{T}$ cell response by immature myeloid cells in cancer is mediated by reactive oxygen species," Journal of Immunology, vol. 172, no. 2, pp. 989-999, 2004.

[29] A. Viola and V. Bronte, "Metabolic mechanisms of cancerinduced inhibition of immune responses," Seminars in Cancer Biology, vol. 17, no. 4, pp. 309-316, 2007.

[30] C. A. Corzo, M. J. Cotter, P. Cheng et al., "Mechanism regulating reactive oxygen species in tumor-induced myeloidderived suppressor cells," Journal of Immunology, vol. 182, no. 9, pp. 5693-5701, 2009.

[31] J. I. Youn, S. Nagaraj, M. Collazo, and D. I. Gabrilovich, "Subsets of myeloid-derived suppressor cells in tumor-bearing mice," Journal of Immunology, vol. 181, no. 8, pp. 5791-5802, 2008.

[32] T. F. Greten, M. P. Manns, and F. Korangy, "Myeloid derived suppressor cells in human diseases," International Immunopharmacology, vol. 11, no. 7, pp. 802-807, 2011.

[33] S. Nagaraj, M. Collazo, C. A. Corzo et al., "Regulatory myeloid suppressor cells in health and disease," Cancer Research, vol. 69, no. 19, pp. 7503-7506, 2009.

[34] M. Kmieciak, J. K. Morales, J. Morales, E. Bolesta, M. Grimes, and M. H. Manjili, "Danger signals and nonself entity of tumor antigen are both required for eliciting effective immune responses against HER-2/neu positive mammary carcinoma: implications for vaccine design," Cancer Immunology, Immunotherapy, vol. 57, no. 9, pp. 1391-1398, 2008.

[35] S. Moulis and D. C. Sgroi, "Re-evaluating early breast neoplasia," Breast Cancer Research, vol. 10, no. 1, article 302, 2008.

[36] S. R. Lakhani, "The transition from hyperplasia to invasive carcinoma of the breast," The Journal of Pathology, vol. 187, pp. 272-278, 1999.

[37] M. Habibi, M. Kmieciak, L. Graham, J. K. Morales, H. D. Bear, and M. H. Manjili, "Radiofrequency thermal ablation of breast tumors combined with intralesional administration of IL-7 and IL-15 augments anti-tumor immune responses and inhibits tumor development and metastasis," Breast Cancer Research and Treatment, vol. 114, no. 3, pp. 423-431, 2009.

[38] Y. Liu, L. Lai, Q. Chen et al., "MicroRNA-494 is required for the accumulation and functions of tumor-expanded myeloidderived suppressor cells via targeting of PTEN," The Journal of Immunology, vol. 188, no. 11, pp. 5500-5510, 2012.
[39] J. I. Youn, M. Collazo, I. N. Shalova, S. K. Biswas, and D. I. Gabrilovich, "Characterization of the nature of granulocytic myeloid-derived suppressor cells in tumor-bearing mice," Journal of Leukocyte Biology, vol. 91, no. 1, pp. 167-181, 2012.

[40] H. L. Kaufman, D. W. Kim, G. Deraffele, J. Mitcham, R. S. Coffin, and S. Kim-Schulze, "Local and distant immunity induced by intralesional vaccination with an oncolytic herpes virus encoding GM-CSF in patients with stage IIIc and IV melanoma," Annals of Surgical Oncology, vol. 17, no. 3, pp. 718-730, 2010.

[41] C. Meyer, A. Sevko, M. Ramacher et al., "Chronic inflammation promotes myeloid-derived suppressor cell activation blocking antitumor immunity in transgenic mouse melanoma model," Proceedings of the National Academy of Sciences of the United States of America, vol. 108, no. 41, pp. 17111-17116, 2011.

[42] I. Poschke, D. Mougiakakos, J. Hansson, G. V. Masucci, and R. Kiessling, "Immature immunosuppressive CD14 ${ }^{+} \mathrm{HLA}-$ $\mathrm{DR}^{-/ l o w}$ cells in melanoma patients are Stat $3^{\text {hi }}$ and overexpress CD80, CD83, and DC-sign," Cancer Research, vol. 70, no. 11, pp. 4335-4345, 2010.

[43] P. Filipazzi, R. Valenti, V. Huber et al., "Identification of a new subset of myeloid suppressor cells in peripheral blood of melanoma patients with modulation by a granulocytemacrophage colony-stimulation factor-based antitumor vaccine," Journal of Clinical Oncology, vol. 25, no. 18, pp. 25462553, 2007.

[44] H. J. Ko, J. M. Lee, Y. J. Kim, Y. S. Kim, K. A. Lee, and C. Y. Kang, "Immunosuppressive myeloid-derived suppressor cells can be converted into immunogenic APCs with the help of activated NKT cells: an alternative cell-based antitumor vaccine," Journal of Immunology, vol. 182, no. 4, pp. 18181828, 2009.

[45] F. Mussai, C. De Santo, and V. Cerundolo, "Interaction between invariant NKT cells and myeloid-derived suppressor cells in cancer patients: evidence and therapeutic opportunities," Journal of Immunotherapy, vol. 35, no. 6, pp. 449-459, 2012.

[46] A. J. G. Simpson, O. L. Caballero, A. Jungbluth, Y. T. Chen, and L. J. Old, "Cancer/testis antigens, gametogenesis and cancer," Nature Reviews Cancer, vol. 5, no. 8, pp. 615-625, 2005.

[47] P. Van Der Bruggen, C. Traversari, P. Chomez et al., "A gene encoding an antigen recognized by cytolytic $\mathrm{T}$ lymphocytes on a human melanoma," Science, vol. 254, no. 5038, pp. 16431647, 1991.

[48] N. N. Hunder, H. Wallen, J. Cao et al., "Treatment of metastatic melanoma with autologous $\mathrm{CD}^{+} \mathrm{T}$ cells against NY-ESO-1," New England Journal of Medicine, vol. 358, no. 25, pp. 2698-2703, 2008.

[49] F. Meklat, Z. Li, Z. Wang et al., "Cancer-testis antigens in haematological malignancies," British Journal of Haematology, vol. 136, no. 6, pp. 769-776, 2007.

[50] A. Mischo, B. Kubuschok, K. Ertan et al., "Prospective study on the expression of cancer testis genes and antibody responses in 100 consecutive patients with primary breast cancer," International Journal of Cancer, vol. 118, no. 3, pp. 696-703, 2006.

[51] T. Badovinac-Crnjevic, G. Spagnoli, A. Juretic et al., "High expression of MAGE-A10 cancer-testis antigen in triplenegative breast cancer," Medical Oncology, vol. 29, no. 3, pp. 1586-1591, 2012.

[52] Y. Sugita, H. Wada, S. Fujita et al., "NY-ESO-1 expression and immunogenicity in malignant and benign breast tumors," Cancer Research, vol. 64, pp. 2199-2204, 2004. 
[53] M. J. Scanlan, A. O. Gure, A. A. Jungbluth, L. J. Old, and Y. T. Chen, "Cancer/testis antigens: an expanding family of targets for cancer immunotherapy," Immunological Reviews, vol. 188, pp. 22-32, 2002.

[54] J. P. Theurillat, F. Ingold, C. Frei et al., "NY-ESO-1 protein expression in primary breast carcinoma and metastases Correlation with $\mathrm{CD}^{+} \mathrm{T}$-cell and CD79a+ plasmacytic/B-cell infiltration," International Journal of Cancer, vol. 120, no. 11, pp. 2411-2417, 2007.

[55] F. Fang, C. Balch, J. Schilder et al., "A phase 1 and pharmacodynamic study of decitabine in combination with carboplatin in patients with recurrent, platinum-resistant, epithelial ovarian cancer," Cancer, vol. 116, no. 17, pp. 4043-4053, 2010.

[56] J. U. Guo, Y. Su, C. Zhong, G. L. Ming, and H. Song, "Emerging roles of TET proteins and 5-hydroxymethylcytosines in active DNA demethylation and beyond," Cell Cycle, vol. 10, no. 16, pp. 2662-2668, 2011.

[57] A. Bird, "DNA methylation patterns and epigenetic memory," Genes and Development, vol. 16, no. 1, pp. 6-21, 2002.

[58] T. Ushijima, "Detection and interpretation of altered methylation patterns in cancer cells," Nature Reviews Cancer, vol. 5, no. 3, pp. 223-231, 2005.

[59] M. L. Ascierto, M. Kmieciak, M. O. Idowu et al., "A signature of immune function genes associated with recurrence-free survival in breast cancer patients," Breast Cancer Research and Treatment, vol. 131, no. 3, pp. 871-880, 2012.

[60] S. M. Pulukuri and J. S. Rao, "Activation of p53/p21Waf1/Cip1 pathway by 5 -aza-2'-deoxycytidine inhibits cell proliferation, induces pro-apoptotic genes and mitogen-activated protein kinases in human prostate cancer cells," International Journal of Oncology, vol. 26, no. 4, pp. 863-871, 2005.

[61] D. Yang, C. M. Torres, K. Bardhan, M. Zimmerman, T. L. McGaha, and K. Liu, "Decitabine and vorinostat cooperate to sensitize colon carcinoma cells to fas ligand-induced apoptosis in vitro and tumor suppression in vivo," The Journal of Immunology, vol. 188, no. 9, pp. 4441-4449, 2012.

[62] M. Y. Chen, W. S. Liao, Z. Lu et al., "Decitabine and suberoylanilide hydroxamic acid (SAHA) inhibit growth of ovarian cancer cell lines and xenografts while inducing expression of imprinted tumor suppressor genes, apoptosis, G2/M arrest, and autophagy," Cancer, vol. 117, no. 19, pp. 4424-4438, 2011.

[63] L. Bao, K. Dunham, and K. Lucas, "MAGE-A1, MAGE-A3, and NY-ESO-1 can be upregulated on neuroblastoma cells to facilitate cytotoxic T lymphocyte-mediated tumor cell killing," Cancer Immunology, Immunotherapy, vol. 60, no. 9, pp. 12991307, 2011.

[64] M. Almstedt, N. Blagitko-Dorfs, J. Duque-Afonso et al., "The DNA demethylating agent 5-aza-2'-deoxycytidine induces expression of NY-ESO-1 and other cancer/testis antigens in myeloid leukemia cells," Leukemia Research, vol. 34, no. 7, pp. 899-905, 2010.

[65] A. A. Toor, K. K. Payne, H. M. Chung et al., "Epigenetic induction of adaptive immune response in multiple myeloma: sequential azacitidine and lenalidomide generate cancer testis antigen-specific cellular immunity," British Journal of Haematology, vol. 158, no. 6, pp. 700-711, 2012.

[66] "Re-expression of ER in Triple Negative Breast Cancers," http://clinicaltrials.gov/ct2/show/NCT01194908?term=decitabine+and+breast+cancer\&rank $=1$.

[67] E. J. Geutjes, S. Tian, P. Roepman, and R. Bernards, "Deoxycytidine kinase is overexpressed in poor outcome breast cancer and determines responsiveness to nucleoside analogs," Breast Cancer Research and Treatment, vol. 131, no. 3, pp. 809-818, 2012.
[68] A. Truneh, F. Albert, P. Golstein, and A. M. Schmitt-Verhulst, "Early steps of lymphocyte activation bypassed by synergy between calcium ionophores and phorbol ester," Nature, vol. 313, no. 6000, pp. 318-320, 1985.

[69] T. M. Tuttle, T. H. Inge, K. P. Bethke, C. W. McCrady, G. R. Pettit, and H. D. Bear, "Activation and growth of murine tumor-specific T-cells which have in vivo activity with bryostatin 1," Cancer Research, vol. 52, no. 3, pp. 548-553, 1992.

[70] T. Chatila, L. Silverman, R. Miller, and R. Geha, "Mechanisms of T cell activation by the calcium ionophore ionomycin," Journal of Immunology, vol. 143, no. 4, pp. 1283-1289, 1989.

[71] M. G. Kazanietz, N. E. Lewin, F. Gao, G. R. Pettit, and P. M. Blumberg, "Binding of [26-3H]bryostatin 1 and analogs to calcium-dependent and calcium-independent protein kinase C isozymes," Molecular Pharmacology, vol. 46, no. 2, pp. 374379, 1994.

[72] T. Chatila, L. Silverman, R. Miller, and R. Geha, "Mechanisms of T cell activation by the calcium ionophore ionomycin," Journal of Immunology, vol. 143, no. 4, pp. 1283-1289, 1989.

[73] M. G. Kazanietz, N. E. Lewin, F. Gao, G. R. Pettit, and P. M. Blumberg, "Binding of [26-3H]bryostatin 1 and analogs to calcium-dependent and calcium-independent protein kinase C isozymes," Molecular Pharmacology, vol. 46, no. 2, pp. 374379, 1994.

[74] J. K. Morales, M. Kmieciak, K. L. Knutson, H. D. Bear, and M. H. Manjili, "GM-CSF is one of the main breast tumor-derived soluble factors involved in the differentiation of CD11b-Gr1bone marrow progenitor cells into myeloid-derived suppressor cells," Breast Cancer Research and Treatment, vol. 123, no. 1, pp. 39-49, 2010.

[75] M. Gehrmann, J. Marienhagen, H. Eichholtz-Wirth et al., "Dual function of membrane-bound heat shock protein 70 (Hsp70), Bag-4, and Hsp40: protection against radiationinduced effects and target structure for natural killer cells," Cell Death and Differentiation, vol. 12, no. 1, pp. 38-51, 2005.

[76] T. E. Schmid and G. Multhoff, "Radiation-induced stress proteins - the role of heat shock proteins (HSP) in anti- tumor responses," Current Medicinal Chemistry, vol. 19, no. 12, pp. 1765-1770, 2012.

[77] S. Gasser, S. Orsulic, E. J. Brown, and D. H. Raulet, "The DNA damage pathway regulates innate immune system ligands of the NKG2D receptor," Nature, vol. 436, no. 7054, pp. 11861190, 2005.

[78] L. Apetoh, F. Ghiringhelli, A. Tesniere et al., "Toll-like receptor 4-dependent contribution of the immune system to anticancer chemotherapy and radiotherapy," Nature Medicine, vol. 13, no. 9, pp. 1050-1059, 2007.

[79] H. Fang, Y. Wu, X. Huang et al., “Toll-like receptor 4 (TLR4) is essential for Hsp70-like protein 1 (HSP70L1) to activate dendritic cells and induce Th1 response," The Journal of Biological Chemistry, vol. 286, no. 35, pp. 30393-30400, 2011.

[80] J. Y. Kim, Y. O. Son, S. W. Park et al., "Increase of NKG2D ligands and sensitivity to NK cell-mediated cytotoxicity of tumor cells by heat shock and ionizing radiation," Experimental and Molecular Medicine, vol. 38, no. 5, pp. 474-484, 2006.

[81] J. A. Aguirre-Ghiso, "Models, mechanisms and clinical evidence for cancer dormancy," Nature Reviews Cancer, vol. 7, no. 11, pp. 834-846, 2007.

[82] A. F. Chambers, A. C. Groom, and I. C. MacDonald, "Dissemination and growth of cancer cells in metastatic sites," Nature Reviews Cancer, vol. 2, no. 8, pp. 563-572, 2002.

[83] P. Goss, A. L. Allan, D. I. Rodenhiser, P. J. Foster, and A. F. Chambers, "New clinical and experimental approaches 
for studying tumor dormancy: does tumor dormancy offer a therapeutic target?" Acta Pathologica, Microbiologica et Immunologica, vol. 116, no. 7-8, pp. 552-568, 2008.

[84] T. G. Karrison, D. J. Ferguson, and P. Meier, "Dormancy of mammary carcinoma after mastectomy," Journal of the National Cancer Institute, vol. 91, no. 1, pp. 80-85, 1999.

[85] N. Almog, "Molecular mechanisms underlying tumor dormancy," Cancer Letters, vol. 294, no. 2, pp. 139-146, 2010.

[86] H. A. Coller, L. Sang, and J. M. Roberts, "A new description of cellular quiescence," PLoS Biology, vol. 4, no. 3, pp. 0329-0349, 2006.

[87] R. Pelayo, K. Miyazaki, J. Huang, K. P. Garrett, D. G. Osmond, and P. W. Kincade, "Cell cycle quiescence of early lymphoid progenitors in adult bone marrow," Stem Cells, vol. 24, no. 12, pp. 2703-2713, 2006. 


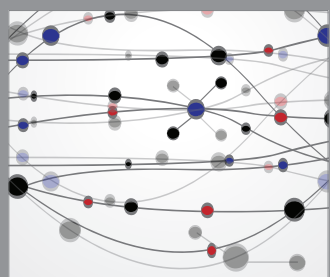

The Scientific World Journal
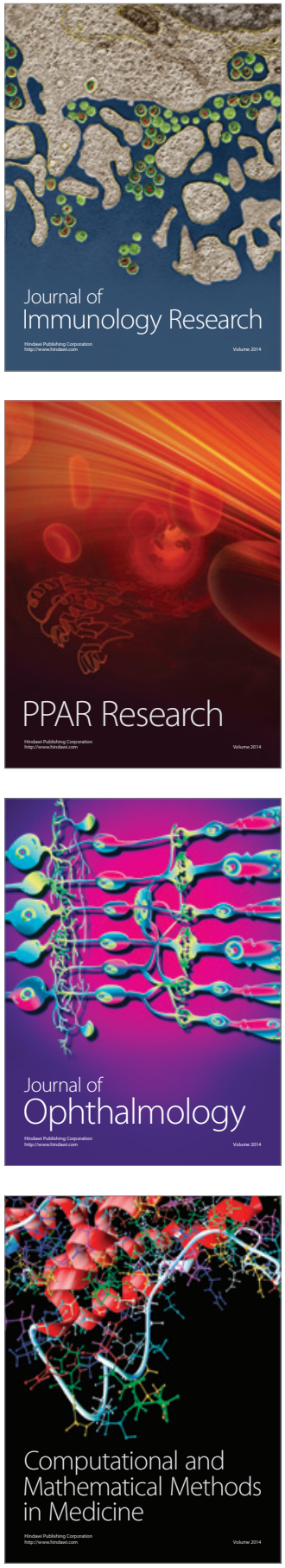

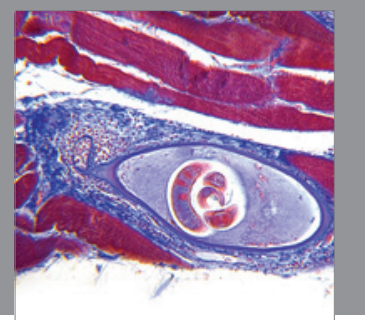

Gastroenterology

Research and Practice
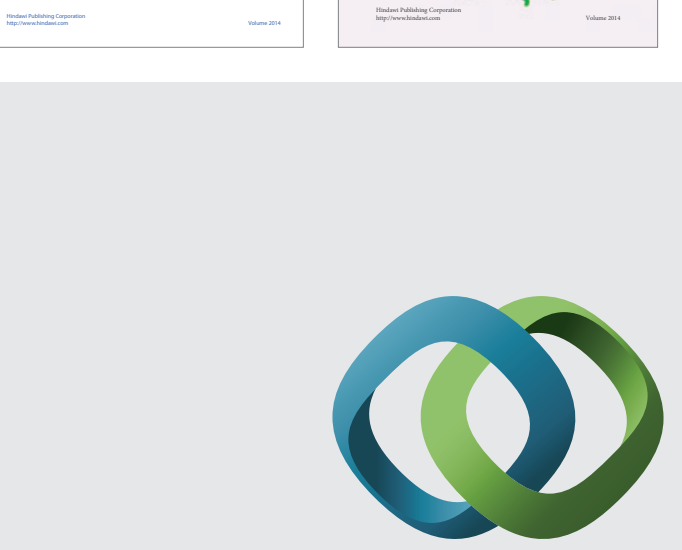

\section{Hindawi}

Submit your manuscripts at

http://www.hindawi.com
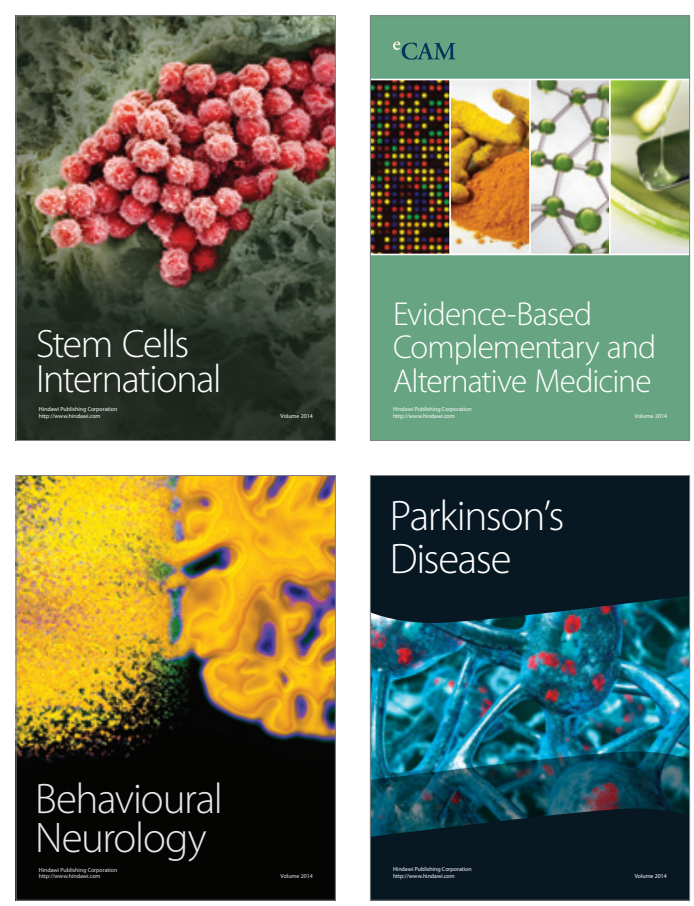

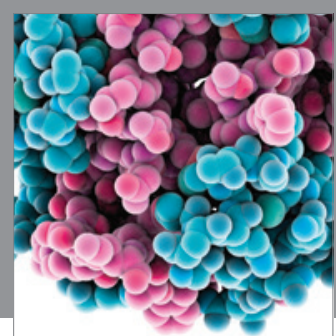

Journal of
Diabetes Research

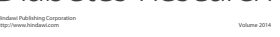

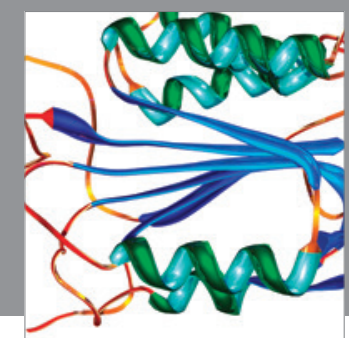

Disease Markers
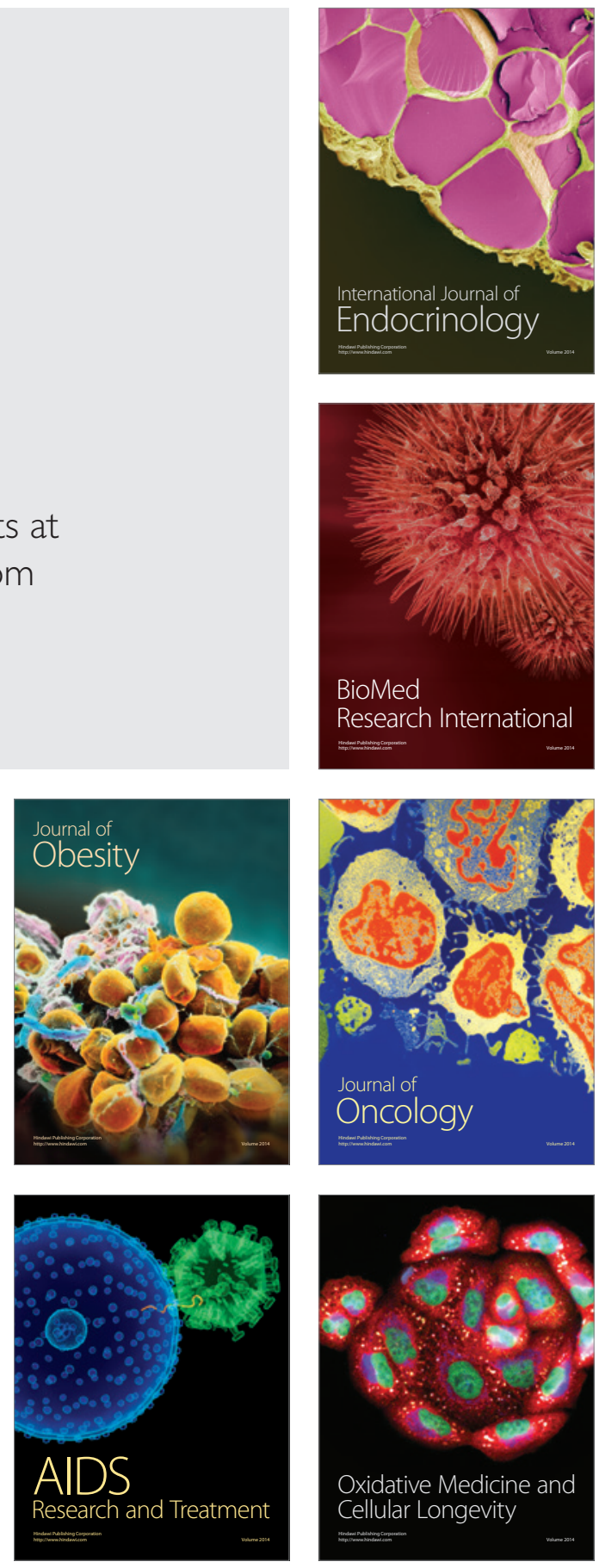